\title{
Newest models and calculation schemes for quantitative analysis of physical properties of polymers
}

\author{
Andrey Askadskii ${ }^{1,2}$, Tatyana Matseevich ${ }^{1, *}$ and Andrey Matseevich ${ }^{1,2}$ \\ ${ }^{1}$ Moscow State University of Civil Engineering, Yaroslavskoe shosse, 26, Moscow, 129337, Russia \\ ${ }^{2}$ A.N. Nesmeyanov Institute of Organoelement Compounds Russian Academy of Sciences, \\ Vavilova str., 28, Moscow, Russia
}

\begin{abstract}
New models and calculation schemes have been developed for the quantitative analysis of a number of physical properties of polymers glass transition temperature, flow temperature of polymer nanocomposites, thermal conductivity, boiling point of polymer solutions, water absorption and water permeability of polymers and nanocomposites, strength, viscosity, storage and losses moduli, refractive index and dielectric constant. All calculation schemes are based on the structure of linear and cross-linked polymers; their degree of crystallinity, free volume, the effect of temperature, the composition of copolymers and homogeneous mixtures of polymers, the concentration of nanoparticles, their shape, size distribution, orientation angles, the structure of polar groups grafted to the surface of nanoparticles, the energy of intermolecular interactions are taken into account. All computational schemes are computerized and allow calculations to be carried out automatically after the introduction of the structure of a repeating unit of polymer unit into the computer, as well as the shape and size of nanofillers.
\end{abstract}

\section{Introduction}

In recent years, we have developed new and modified previous calculation schemes for the quantitative description of a number of physical properties of polymers. Among the properties are the glass transition temperature, the flow temperature of polymer nanocomposites, thermal conductivity, boiling point of polymer solutions, water absorption and water permeability of polymers and nanocomposites, the strength, viscosity, storage and losses moduli, refractive index and dielectric constant. All design schemes are based on the structure of linear and cross-linked polymers; their degree of crystallinity, free volume, temperature effect, composition of copolymers and homogeneous mixtures of polymers are taken into account. The concentration of nanoparticles, their shape, size distribution, orientation angles, the structure of polar groups grafted to the surface of the nanoparticles, the energy of intermolecular interactions are taken into account. Spherical nanoparticles, rectangular plates and nanofibers are considered. The calculation scheme for the refractive

\footnotetext{
Corresponding author: MatseevichTA@mgsu.ru
} 
index and dielectric constant takes into account the influence of the plasticizing effect of the residues of the synthesis products and the solvent, nonlinearity on the Clausis-Mossoty function, the composition of nanoparticles, and temperature. All computational schemes are computerized and allow calculations to be carried out automatically after the introduction of the structure of a repeating unit of polymer into the computer, as well as the shape and size of nanofillers.

\section{Methods}

Used methods for constructing models and design schemes, published in [1-5]. To develop computational schemes, it is necessary to calculate the van der Waals volume of repeating units of polymers, as well as the energy of intermolecular interactions. In the calculations of the refractive index and dielectric constant, molar refraction and polarizability were calculated. In the work actively used a computer program "Cascade", developed in INEOS RAS.

\section{Results}

\subsection{Glass transition temperature of polymers}

In order to modify the calculation scheme [1-3] to estimate the glass transition temperature, we introduced atomic constants and energies of dipole - dipole interactions and hydrogen bonds for atoms and atomic groups located in the main and side chains. The equation used to describe the glass transition temperature of polymers $T_{g}$ :

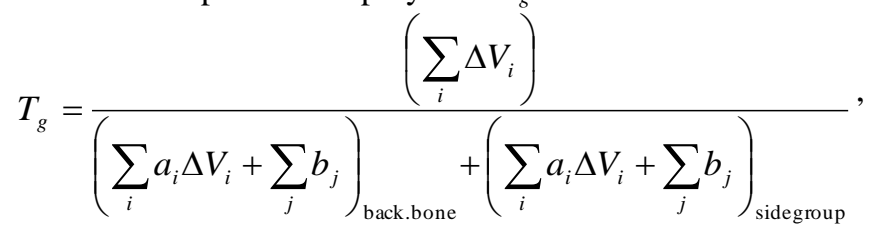

where $a_{i}$ are the atomic constants associated with the energy of weak dispersion interaction; $b_{j}$ are the constants associated with the energy of the dipole-dipole interactions of $b_{d}$ and hydrogen bonds $b_{h} ;\left(\sum_{i} \Delta V_{i}\right)$ is the van der Waals volume of the repeating unit.

The correlation diagram containing over 50 polymers is shown in Figure 1; the correlation coefficient is 0.999 .

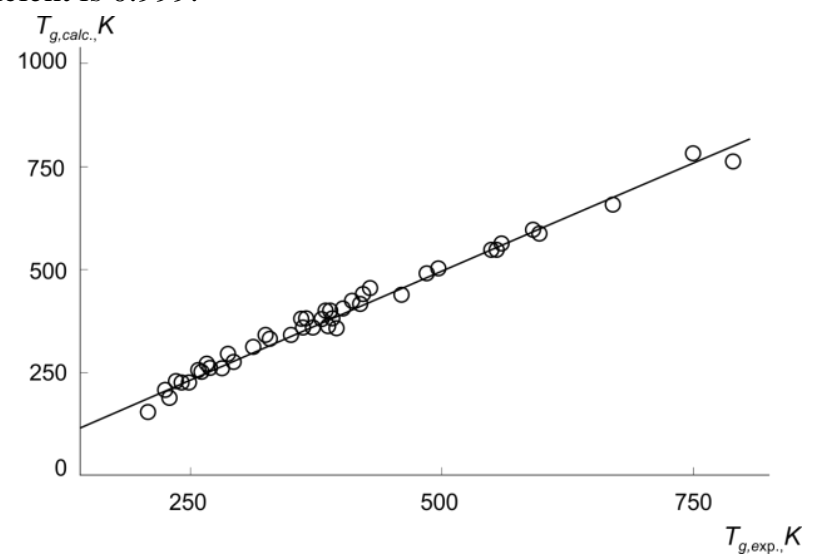

Fig. 1. Correlation diagram. $T_{g \text {,exp. }}$ and $T_{g, \text { calc }}$. are the experimental and calculated values of glass transition temperature, respectively. 


\subsection{Flow temperature of polymer nanocomposites}

Flow temperature $T_{f}$ of polymer nanocomposites is calculated by the ratio, which includes the concentration and radius of the nanoparticles, the number of polar groups grafted on their surface, the density of the nanoparticles, the molecular weight of the polymer.

\subsection{Thermal conductivity of polymers}

This characteristic in this paper is calculated by the formula

$$
\lambda=\frac{\sum_{i} a_{i}+\sum_{j} b_{j}}{N_{A} \sum_{i} \Delta V_{i}} \cdot \frac{m^{1 / 3} \cdot c_{p} \cdot \rho^{4 / 3}}{M_{0}^{1 / 3}},
$$

where $a_{i}$ are the atomic constants characteristic of each atom; $b_{j}$ are the constants for polar groups leading to dipole-dipole interaction or to hydrogen bonds; $N_{A}$ is the Avogadro number, $\Delta V_{i}$ is the van der Waals volume of the $i$-th atom, $m$ is the number of atoms in the repeating unit, $c_{p}$ is the specific heat capacity, $M_{0}$ is the molecular mass of the repeating unit, $\rho$ is the density.

The arrangement of atoms in the back bone and side chains, the degree of crystallinity are taken into account. The correlation diagram is shown in Figure 2.

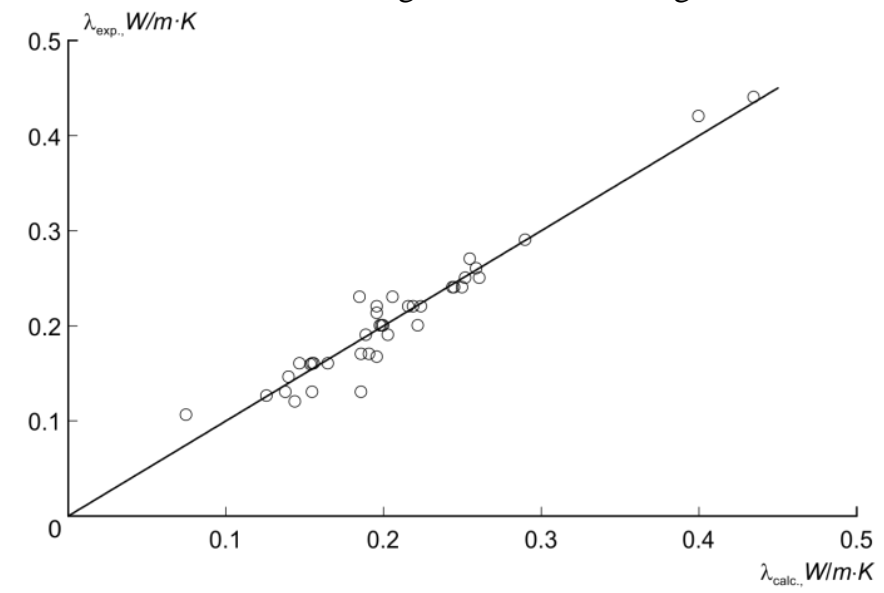

Fig. 2. Correlation diagram for the set of amorphous and semi-crystalline polymers

\subsection{Calculation scheme for estimating the boiling point of a mixture of polymer and solvent.}

This design scheme is important for the ebullioscopic method for determining the molecular masses of polymers. The enthalpy of evaporation of the solvent at the boiling point is expressed in terms of the Hildebrand solubility parameter, which, in turn, depends on the temperature. As a result, the ratio was obtained linking the boiling point of the polymer solution with the mole and weight fractions of the dissolved polymer:

$$
T_{1}=\frac{T_{0}}{R T_{0} \ln \left(1-\frac{1}{1+\frac{M_{p}}{M_{s}}\left(\frac{1}{\alpha_{w, p}}-1\right)}\right)},
$$


where $T_{0}$ is the boiling point of the solvent, $T_{1}$ is the boiling point of the solution, $\delta$ is the solubility parameter (Hildebrand parameter), $R$ is the universal gas constant, $V$ is the molar liquid volume, $M_{p}$ and $M_{s}$ are the molecular masses of the polymer and solvent, respectively, $\alpha_{w, p}$ is the weight fraction of polymer.

The proposed calculation scheme for quantitative estimation of the boiling point of polymer solutions in an organic solvent allows calculations based on the chemical structure of the polymer and solvent, does not require preliminary experiments, and therefore has predictive power.

\subsection{Water absorption and water permeability of polymers and copolymers}

The correlation is proposed for calculating the activation energy of the permeability process, based on the set of atomic constants describing the contribution of each atom and polar groups to the energy of intermolecular interaction with water, which are summarized to estimate the total energy. The chemical structure of the polymer, the degree of crystallinity, temperature and free (empty) volume are taken into account.

$$
\ln P=\ln P_{0}-\frac{\sum_{i} \Delta E_{i}^{* *}}{N_{A} R T \sum_{i} \Delta V_{i}},
$$

where $P$ is the permeability, $P_{0}$ is the constant, $\sum_{i} \Delta V_{i}$ is the van der Waals volume of the repeating unit of polymer, $\sum_{i} \Delta E_{i}^{* *}$ is the activation energy of the permeability process multiplied by the van der Waals volume of the repeating unit.

The method is computerized. The computer program allows to solve both the direct problem associated with the evaluation of the properties of polymers based on the chemical structure of the repeating unit, and the inverse problem, which consists in searching for such polymer structures that have a given permeability interval.

\subsection{Design scheme for assessing the permeability of nanocomposites}

The calculation scheme has been developed for predicting water permeability through polymer nanocomposites. The chemical structure of the polymer and its concentration, the chemical structure of the nanoparticle surface, the concentration of nanoparticles in the composite and the concentration of polar functional groups on the surface of the nanoparticles, the size and shape of nanoparticles are taken into account. The concentration of nanoparticles and the number of polar groups on their surface have the greatest effect on permeability. The influence of the size distribution of anisometric nanoparticles and their orientation on permeability is analyzed. The calculation method is computerized and included as the special option in the CASCADE computer program (INEOS RAS), which allows calculations and predictions of the permeability of nanocomposites after the chemical structure and the specified parameters of nanoparticles are introduced into the computer.

The permeability of the composite $P$, consisting of a polymer and nanoparticles, is described by the ratio

$$
P=P_{0}^{*}\left(1-\alpha_{d, f}\right)
$$

where $P_{0}^{*}$ is the permeability of the original polymer, $\alpha_{d, f}$ is the volume fraction of nanoparticles. 
For nanocomposites, in which the surface of the nanoparticles is modified by chemical groups of a particular chemical structure, the following relationship applies:

$$
\ln P_{0}^{*}=\frac{\frac{1}{1+\beta}\left[\left(\sum_{i} \Delta E_{i}^{* *}\right)_{p}-\left(\sum_{i} \Delta E_{i}^{* *}\right)_{c g}\right]+\left(\sum_{i} \Delta E_{i}^{* *}\right)_{c g}}{N_{A}\left\{\frac{1}{1+\beta}\left[\left(\sum_{i} \Delta V_{i}\right)_{p}-\left(\sum_{i} \Delta V_{i}\right)_{c g}\right]+\left(\sum_{i} \Delta V_{i}\right)_{c g}\right\}},
$$

where $\left(\sum_{i} \Delta E_{i}^{* *}\right)_{p}$ and $\left(\sum_{i} \Delta E_{i}^{* *}\right)_{c g}$ are the reduced values of the energy of intermolecular interaction for the repeating unit of the polymer and for the chemical group localized on the surface of the nanoparticle, respectively: $\left(\sum_{i} \Delta V_{i}\right)_{p}$ and $\left(\sum_{i} \Delta V_{i}\right)_{c g}$ are the van der Waals volumes of the repeating unit of the polymer and chemical group located on the surface of the nanoparticle, respectively.

The value of $\beta$ is described by the formula

$$
\beta=\frac{n_{c g} c_{n p} M_{0}}{\rho_{n p} v_{n p} c_{p} 0.6022 \cdot 10^{24}},
$$

where $n_{c g}$ is the number of polar groups on the surface of one nanoparticle, $c_{n p}$ is the weight of nanoparticles in a nanocomposite, $c_{p}$ is the weight of the polymer, $\rho_{n p}$ is the density of the nanoparticle, $v_{n p}$ is the volume of one nanoparticle.

\subsection{Analysis of the influence of the chemical composition and concentration of the components of the polymer-solvent mixture on the strength and viscosity}

The calculation scheme is proposed for estimating the strength $\sigma_{y}$ and viscosity $\eta$ of a system containing a polymer and a solvent (plasticizer). This scheme takes into account the chemical structure of the polymer and the solvent, the intermolecular interaction between the polymer and the solvent, the van der Waals volume and molecular weight of the repeating unit of the polymer and the solvent molecule, as well as the concentration of the solvent.

Since the value of strength is measured in the glassy state of the system (in our case, in the polymer + solvent system), the following relationship applies:

$$
\sigma_{y}=\frac{\left(1-\alpha_{m, s+p}\right)\left(\sum_{i} \Delta V_{i}\right)_{p}+\alpha_{m, s+p}\left(\sum_{i} \Delta V_{i}\right)_{s+p}}{\left(1-\alpha_{m, s+p}\right) \frac{\left(\sum_{i} \Delta V_{i}\right)_{p}}{\sigma_{y, p}}+\alpha_{m, s+p} \frac{\left(\sum_{i} \Delta V_{i}\right)_{s+p}}{\sigma_{y, s+p}}},
$$

where $\alpha_{m, s+p}$ is the mole fraction of the polymer + solvent mixture, $\left(\sum_{i} \Delta V_{i}\right)_{s+p}$ is van der

Waals volume of the mixture mentioned above, which is critical (if the concentration of solvent exceeds the critical one, the mixture goes into a rubbery state); $\sigma_{y, s+p}$ is the strength for the "polymer + solvent" system, which has a critical solvent concentration. 
The final equation for calculating the viscosity depending on the volume fraction of the polymer is

$$
\ln \left(\frac{\eta}{B}\right)=4 \ln c_{p} \alpha_{v, p}+\left\{\alpha_{v, p}\left[0.025+\left(\alpha_{L}-\alpha_{G}\right)\left(T-T_{g, p}\right)\right]+\left(1-\alpha_{v, p}\right)\left[0.025+\alpha_{s}\left(T-T_{g, s}\right)\right]\right\}^{-1},(9)
$$

where $B$ is a combined constant, including molecular weight; $c_{p}$ is the polymer concentration, $\alpha_{v, p}$ is the volume fraction of the polymer, $T$ is the temperature, $T_{g, p}$ is the glass transition temperature of the polymer, $\alpha_{\mathrm{s}}$ is the thermal expansion coefficient of the solvent (plasticizer), $T_{g, s}$ is the glass transition temperature of the solvent, $\alpha_{L}$ and $\alpha_{G}$ are the thermal expansion coefficients polymer in the rubbery and glassy state of the polymer, respectively.

This calculation scheme allows us to predict the dependence of the strength and viscosity of the "polymer + plasticizer" system.

\subsection{The calculation scheme for estimating the dynamic viscosity, storage modulus and loss modulus depending on temperature}

In [4], it was shown that the storage modulus of polymer at high frequencies is described by the following equation

$$
G=\frac{\sum_{i} \Delta V_{i}}{\sum_{i} g_{i} \Delta V_{i}+\sum_{j} f_{j}},
$$

where $g_{i}=\frac{S_{i}}{\kappa_{i} l_{i}}$; the $g_{i}$ values characterize the average contribution of each atom to the quantity $\frac{S_{i}}{\kappa_{i} l_{i}} ; f_{i}$ is the set of constants that characterize the effect of a strong intermolecular interaction (dipole-dipole interaction, hydrogen bonds); $S_{i}$ is the van der Waals surface of the $i$-th atom, through which the intermolecular interaction is transferred; $\kappa_{i}$ is the coefficient of elasticity of the bond of the $i$-th atom; $l_{i}$ is the characteristic bond size; $\Delta V_{i}$ is the van der Waals volume of the $\mathrm{i}$-th atom included in the repeating unit of the polymer.

The values of $g_{i}$ and $f_{j}$ found in [5] are shown in table 1:

Table 1. The values of the constants $g_{i}$ and $f_{j}$.

\begin{tabular}{|l|c|c|}
\hline \multicolumn{1}{|c|}{$\begin{array}{c}\text { Atom or type of } \\
\text { intermolecular interaction }\end{array}$} & \multicolumn{2}{c|}{ Values $g_{i}$ or $f_{j}$} \\
\hline Carbon & $g_{C}, \mathrm{~cm}^{2} / \mathrm{kg}$ & -1.277 \\
\hline Hydrogen & $g_{H}, \mathrm{~cm}^{2} / \mathrm{kg}$ & 4.376 \\
\hline Oxygen & $g_{O}, \mathrm{~cm}^{2} / \mathrm{kg}$ & 116.95 \\
\hline Double bond & $f_{\neq}, \AA^{3} \cdot \mathrm{cm}^{2} / \mathrm{kg}$ & 30.925 \\
\hline Dipole-dipole interaction* & $f_{d}, \AA^{3} \cdot \mathrm{cm}^{2} / \mathrm{kg}$ & 84.472 \\
\hline
\end{tabular}

$* f_{d}$ parameter for each branch in the back bone or side chain.

\section{Conclusions}

Thus, the proposed and modified computational schemes make it possible to accurately evaluate various physical characteristics of polymers and polymeric materials based on their chemical structure, composition of mixtures and nanocomposites, and the nature of the orientation of nanoparticles. The influence of the structure of polar groups grafted onto the surface of nanoparticles and their size distribution are also taken into account. The design 
schemes described in this article can provide substantial assistance in the development of new building polymeric materials.

This work was financially supported by Ministry of Science and Higher Education of the Russian Federation (\#NSh-3492.2018.8).

\section{References}

1. A.A. Askadskii, Computational Materials Science of Polymers (CISP, 2003)

2. A.A. Askadskii, Physical Properties of Polymers, Prediction and Control (Gordon and Breach Publishers 1996)

3. A.A. Askadskii, V.I. Kondrashchenko, Komp'yuternoe materialovedenie polimerov (Nauch. Mir 1999)

4. T.A. Matseevich, Relaksatsionnye $i$ bar'yernye svoystva polimernykh materialov stroitel'nogo naznacheniya (2017)

5. A.A. Askadskii AA, T.A. Matseevich, V.I. Kondrashchenko, Plasticheskie massy, 1112, (to be published 2018) 